$\xi_{\mathrm{P}}$

\title{
Smart Cluster Communication Integration and Architecture
}

\author{
Rajesh Babu Natha ${ }^{*}$, K.Sreenivasa Ravi ${ }^{2}$ \\ ${ }^{1}$ Research Scholar, ECE Department, ${ }^{2}$ Professor, ECM Department, Koneru Lakshmaiah Education Foundation, \\ Green Fields, Vaddeswaram, Guntur, Andhra Pradesh, India 522502 \\ *Corresponding Author Email: rajeshnatha@gmail.com
}

\begin{abstract}
In the present day modern world, most of the people are making use of the technology in many ways which made man feasible to complete his tasks at very low time. In this aspect of digitalization, almost all the levels of the society is able to use the services of modern technology like wireless communication, Information Storage and transfer. In this aspect, an emerging medium for smart solution is LPWAN. It uses LoRa communication protocol for Information Interchange. This paper deals with the architecture of smart clusters of cities and solutions to some problems like Public safety, Pollution, Traffic management, Parking Solutions.
\end{abstract}

Keywords: Smart City, Wireless Networks, Information and Communication, LPWAN.

\section{Introduction}

Recent trends in providing Machine to Machine (M2M) networking for nearby equipment or devices for data transfer has brought a vast change. An area under these drastic changes is smart cluster regions in which Information and Communication Technology (ICT) has brought people dwelling in smart areas to access many services. One such major development that happened is Internet of Things (IoT). IoT means devices or objects which interact individually with each other under standard specified protocols [1]. In M2M data transfer, multiple number of technologies were available suitable for different point of use. For data transfer to nearby machine, where 'nearby' could be around hundreds of meters, network of machines can be achieved by using Bluetooth, Zigbee or short range enabled technologies. In sequence to IoT, expansions in mobile communications over the world around last few years were huge and its usage to millions of users [2]. Depending on the vast development of technology, data storage and data transfer has increased in large scale. Present day mobile device market has more capacity to store and exchange. Due to these abilities, we have a vast scope in developing applications which can monitor surroundings of the person or user living place. Challenges among these abilities is to collect, transfer and store information from different sources. Wireless networks has deployed in this stream to analyze large sets of data.

Smart clusters are regions which use ICT like WSN, RFID and mobile communications as sensing component to hold infrastructure and wireless services [3]. To transfer sources data effectively, LPWAN was a suggested protocol and a low power broad band convergence network for IoT. LPWAN solves the problem of complex smart home gateway.
In this brief, we focus on smart cluster regions and its problem of public safety which uses LoRa, one of the promising wide areas Internet of Things Technologies [4].

The smart cluster deployments include Traffic Management, Public Safety, Pollution Monitoring and Parking Problems. The later describes the enabling technologies for smart clusters and the architecture of the system for Information Interchange.

\section{Overview of LPWAN}

Recent trends on developments in communication to support Internet of Things has given rise to LPWAN-Low Power Wide Area Network. This Technology promises to support long-range, low-power consumption, low cost for both the devices and infrastructure, and connect a massive number of devices.

Low Power Wide Area (LPWA) networks depict a view of the present day communication, which will accompany modern cellular and short range wireless technologies in addressing diverse requirements of IoT applications. A Low Power technology delivers some features including wide connectivity for low power and low data rate devices. The scope of this technology is expected to be very vast.

\section{A. Low-Power}

IoT devices are expected to operate for a very long time (several years) without the need to replace the battery. LPWANs achieve low power operation using several approaches. First, they usually form a star topology, which eliminates the energy consumed through packet routing in multihop networks. Second, they keep the node design simple by offloading the complexities to the BS/gateway. Third, they use narrowband channels, decreasing the noise-level and extending the transmission range. 


\section{B. Low Operational Cost}

A major factor to the rise of LPWANs is its low cost. Non-cellular LPWANs require no (or limited) infrastructure and operate on unlicensed spectrum, providing an excellent alternative to the cellular network. In addition, the advances in the hardware design and the simplicity of LPWAN end-devices makes LPWANs economically viable.

\section{Robustness}

LPWANs are designed to provide reliable and robust communications. Most LPWANs adopt robust modulation techniques and spread-spectrum techniques to increase the signal resistance to interference and provide a level of security. In spreadspectrum, narrowband signal is spread in the frequency domain with the same power density resulting in a wider bandwidth signal.

\section{Scope to Scale}

Avoidance of multihop topology gives high potential to scale the LPWANs. In addition, LPWANs use narrowband to support a massive number of devices to efficiently utilize the limited spectrum. Besides some LPWANs, it uses multiple antenna systems to enable the BS to support large number of nodes. Some adopts massively parallel communications in both directions using single antenna system (e.g., SNOW), thus providing opportunities to scale. Scalability of LPWAN is also affected by a number of factors such as the underlying MAC (media access control) protocol, duty-cycle, and reliability requirement.

\section{E. Long-Range Connectivity}

In contrast to traditional short-range wireless sensor networks, the design goal of LPWANs is to offer wide-area coverage at lowpower, and low cost. Most LPWANs achieve long communication range and thus form a star topology where the devices directly communicate with the base station (BS). LPWANs operate on low frequencies (sub-GHz band) that provide long communication range (from few kilometers in urban areas to tens of kilometers in rural areas). Lower frequencies have better propagation characteristic through obstacles. These properties made sub-GHz band attractive for LPWANs technologies.

\section{System Model}

The model which can collaborate the fulfillment of requirements and its deliverables is the target aspects in smart city clusters. Many of the works related to smart cluster design has originated from the WSN roots [3]. This also has a large interlinked dependency between different models of a smart cluster, which puts complexity to data transfer.

One of our development models in Smart Clusters is the issue related to Public. Public Safety issues and their solutions are our proposed system design. Our model uses a safety wearable device for the public. This model uses LoRa Technology for Information Interchange and the LoRa Stack is shown in Fig.1

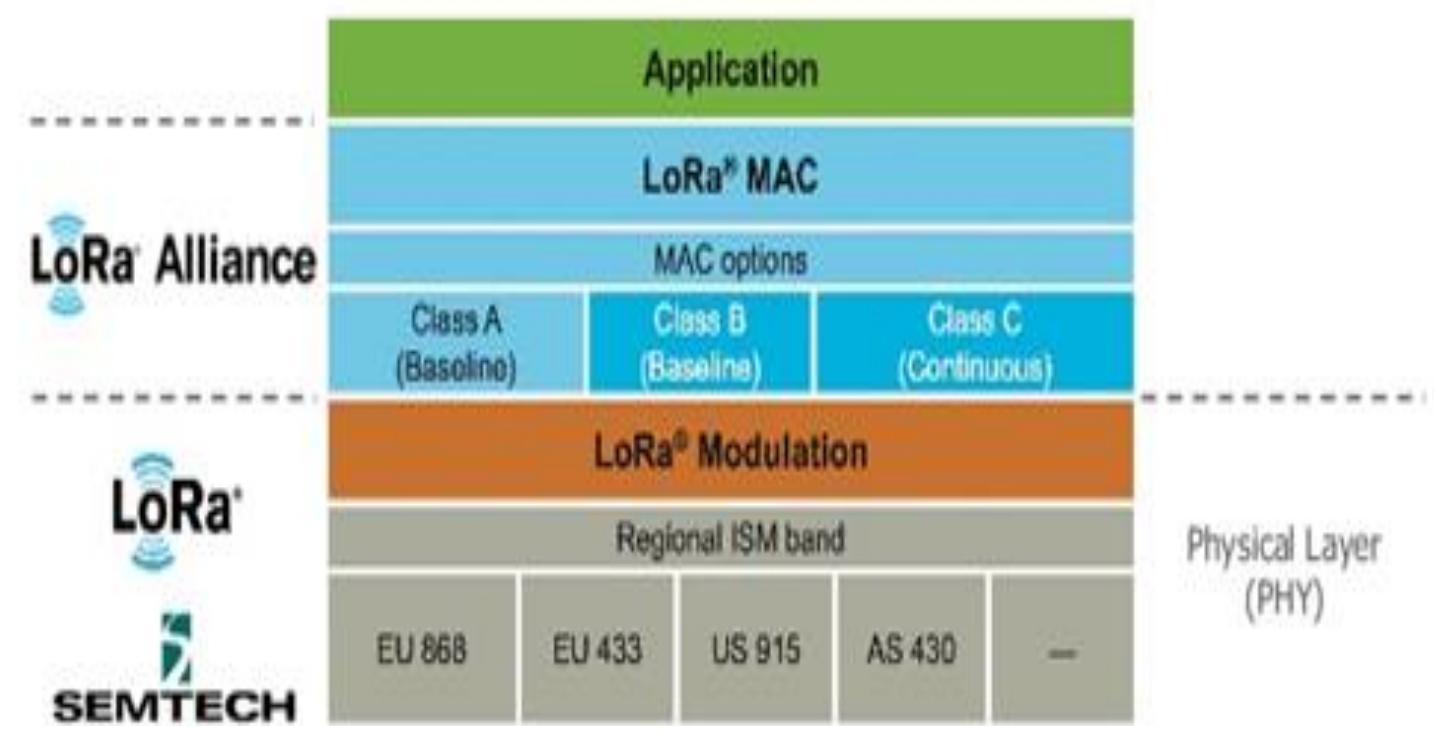

Fig. 1: LoRa Stack

LoRa is the Physical Layer of OSI model for wireless Modulation to create long range communication. It is a proprietary of Semtech based on Chirp Spread Spectrum Modulation. This architecture relies on three layer model. These involve Sensing, Network and Control Layers. In our model, we have recourses for sensing in three forms. These will be WSN, RFID and crowd-sourcing. Wireless Networks take the predominant role in sensing layer. These networks enable the fetch, process and analysis of data in any kind of environment. RF identification provides tagging of objects to monitor them remotely so as to enable the use of it in any kind of application. Depending upon the increase of networks and also the usage of mobile devices, public share their activities and observe in interactive environments [4].

In this proposed model, we link objects to each other for information interchange. The Network layer provides the communication infra to transfer the data from sensing layer to control layer. The main part of our model is the data transfer through internet. In the network view, researches tried to adapt the IoT to IP technology. For instance, 6LoWPAN [5] have followed the approach of reducing the complexity of IP stack to achieve the protocol designed to route IP over everything [1]. In this context, we consider the gateway as a 
link interface between the sensing layer and control layer to modify the protocols used in the sensing layer to IP.

The control layer of this model consists of management of database, knowledge discovery and service management. These areas are the cause for receiving information from data bases and apply filtering algorithms to know new patterns in the data, registering and manage the services which are provided by multiple service providers. This center need not communicate with each sensor individually in order to get data.

\section{Working}

In this brief we have designed a safety wearable for Public Safety. It contains a GPS module and an emergency button. Whenever a person presses this emergency button an alert with Location is send to concerned people. We will create a geo fence for this module. If the device is out of this fence, immediately an alert is generated. The Architecture for this model is shown in Fig-2.

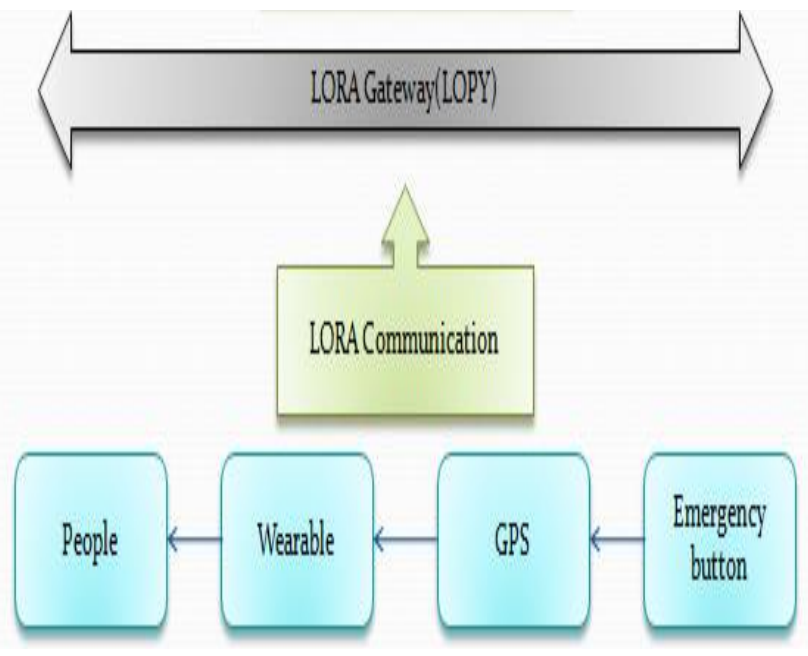

Fig. 2: Public Safety Wearable

The device at a person transfers data like GPS location and button status continuously to the Lora Gateway through Lora communication. This gateway collects the node information and sends to the server or cloud through Wi-Fi communication. Data is stored in the cloud and notifications are generated from the server in emergency cases.

LoRa Gateway is a LOPY nano gateway with Wi-Fi and BLE microcontroller for enterprise grade connected things. It is a Micropython enabled device with super small and light to fit in any cavity. It uses low power to operate or transmit data.

\section{Conclusion}

In this architecture design of Smart Clusters, we have considered Public safety management issue as one of the Cluster issues. We have designed a wearable device for people. It can be worn by any person and the functioning of it explained in brief. This device helps every person of the society providing security and information interchange in the fence created from which it creates the life of the inhabitants easier and smart with security.

\section{References}

[1] L. Atzori, A. Iera, and G. Morabito, "The internet of things: A survey," Computer networks, vol. 54, pp. 2787-2805, 2010.

[2] M. Chen, S. Mao, and Y. Liu, "Big Data: A Survey," Mobile Networks and Applications, vol. 19, pp. 171-209, 2014.

[3] J. Gubbi, R. Buyya, S. Marusic, and M. Palaniswami, "Internet of Things (IoT): A vision, architectural elements, and future directions," Future Generation Computer Systems, vol. 29, pp.1645-1660, 2013.

[4] M. Srivastava, T. Abdelzaher, and B. Szymanski, "Human-centric sensing," Philosophical Transactions of the Royal Society A: Mathematical, Physical and Engineering Sciences, vol. 370, pp. 176197, 2012.

[5] Z. Shelby and C. Bormann, 6LoWPAN: The wireless embedded Internet vol. 43: John Wiley \& Sons, 2011.

[6] Avinash Yadlapati, Dr. Hari Kishore Kakarla, "An Advanced AXI Protocol Verification using Verilog HDL", Wulfenia Journal, ISSN: 1561-882X, Volume 22, Number 4, pp. 307-314, April 2015.

[7] P Ramakrishna, K. Hari Kishore, "Design of Low Power 10GS/s 6Bit DAC using CMOS Technology "International Journal of Engineering and Technology(UAE), ISSN No: 2227-524X, Vol No: 7, Issue No: 1.5, Page No: 226-229, January 2018.

[8] A Murali, K. Hari Kishore, "Efficient and High Speed Key Independent AES Based Authenticated Encryption Architecture using FPGAs "International Journal of Engineering and Technology(UAE), ISSN No: 2227-524X, Vol No: 7, Issue No: 1.5, Page No: 230-233, January 2018.

[9] G.S.Spandana, K Hari Kishore "A Contemporary Approach For Fault Diagnosis In Testable Reversible Circuits By Employing The CNT Gate Library" International Journal of Pure and Applied Mathematics, ISSN No: 1314-3395, Vol No: 115, Issue No: 7, Page No: 537-542, September 2017.

[10] K Hari Kishore, CVRN Aswin Kumar, T Vijay Srinivas, GV Govardhan, Ch Naga Pavan Kumar, R Venkatesh "Design and Analysis of High Efficient UART on Spartran-6 and Virtex-7 Devices", International Journal of Applied Engineering Research, ISSN 0973-4562, Volume 10, Number 09 , pp. 23043-23052, June 2015

[11] K Bindu Bhargavi, K Hari Kishore "Low Power BIST on Memory Interface Logic", International Journal of Applied Engineering Research, ISSN 0973-4562, Volume 10, Number 08 , pp. 2107921090, May 2015.

[12] Korraprolu Brahma Reddy, K Hari Kishore, "A Mixed Approach for Power Dissipation Reduction in Nanometer CMOS VLSI circuits", International Journal of Applied Engineering Research, ISSN 09734562 Volume 9, Number 18, pp. 5141-5148, July 2014.

[13] Nidamanuri Sai Charan, Kakarla Hari Kishore "Reorganization of Delay Faults in Cluster Based FPGA Using BIST" Indian Journal of Science and Technology, ISSN No: 0974-6846, Vol No.9, Issue No.28, page: 1-7, July 2016.

[14] Sravya Kante, Hari Kishore Kakarla, Avinash Yadlapati,"Design and Verification of AMBA AHB-Lite protocol using Verilog HDL" International Journal of Engineering and Technology, E-ISSN No: 0975-4024, Vol No.8, Issue No.2, Page:734-741, April-May 2016.

[15] Bandlamoodi Sravani, K Hari Kishore, "An FPGA Implementation of Phase Locked Loop (PLL)", International Journal of Applied Engineering Research, ISSN 0973-4562, Volume 10, Number 14 , pp. 34137-34139, August 2015.

[16] Avinash Yadlapati, Kakarla Hari Kishore,"Constrained Level Validation of Serial Peripheral Interface Protocol", Proceedings of the First International Conference on SCI 2016, Volume 1, Smart Computing and Informatics, Smart Innovation, Systems and Technologies 77, ISSN No: 2190-3018, ISBN: 978-981-10-5544-7, Chapter No: 77, pp. 743-753, 25 ${ }^{\text {th }}$ December 2017.

[17] P Kiran Kumar, P Prasad Rao, Kakarla Hari Kishore, "Optimal Design of Reversible Parity Preserving New Full Adder / Full Subtractor", IEEE SPONSORED 3rd INTERNATIONAL CONFERENCE ON ELECTRONICS AND COMMUNICATION SYSTEMS (ICECS 2016), pp. $3465-3470,25^{\text {th }}$ and $26^{\text {th }}$ February 2016.

[18] Y Avinash, K Hari Kishore 'Designing Asynchronous FIFO for Low Power DFT Implementation"' International Journal of Pure and 
Applied Mathematics, ISSN No: 1314-3395, Vol No: 115, Issue No: 8, Page No: 561-566, September 2017.

[19] Mahesh Mudavath and K Hari Kishore "Design of RF Front End CMOS Cascade CS Low Noise Amplifier on 65nm Technology Process" International Journal of Pure and Applied Mathematics, ISSN No: 1314-3395, Vol No: 115, Issue No: 7, Page No: 417-422, September 2017.

[20] P. Sahithi K Hari Kishore, E Raghuveera, P. Gopi Krishna "DESIGN OF VOLTAGE LEVEL SHIFTER FOR POWER-EFFICIENT APPLICATIONS USING 45nm TECHNOLOGY" International Journal of Engineering and Technology(UAE), ISSN No: 2227524X, Vol No: 7, Issue No: 2.8, Page No: 103-108, March 2018.

[21] N Bala Dastagiri K Hari Kishore "A 14-bit 10kS/s Power Efficient $65 \mathrm{~nm}$ SAR ADC for Cardiac Implantable Medical Devices" International Journal of Engineering and Technology(UAE), ISSN No: 2227-524X, Vol No: 7, Issue No: 2.8, Page No: 34-39, March 2018.

[22] Shaik Razia, P.Swathi Pryathyusha, N.Vamsi Krishna "A Comparative study of machine learning algorithms on thyroid disease prediction" International Journal of Engineering and Technology(UAE), ISSN No: 2227-524X, Vol No: 7, Issue No: 2.8, Page No: 315-319, March 2018

[23] S RAZIA, A REVIEW ON DISEASE DIAGNOSIS USING MACHINE LEARNING TECHNIQUES, International Journal of Pure and Applied Mathematics, ISSN No: 1314-3395, Vol No: 117, Issue No: 16, Page No: 79-85, 2017

[24] S Razia, MR Narasingarao, GR Sridhar, "A decision support system for prediction of thyroid disease-a comparison of multilayer perception neural network and radial basis function neural network", Journal of Theoretical and Applied Information Technology, ISSN 1992-8645, Volume 80, No. 3 , pp. 544, 2015

[25] SHAIK RAZIA, M.R.Narasingarao,"Development and Analysis of Support Vector Machine Techniques for Early Prediction of Breast Cancer and Thyroid" Journal of Advanced Research in Dynamical and Control Systems, ISSN: 1943-023X, Vol.9.Sp.Issue:6, page no: 869-878, 2017.

[26] S.V.Manikanthan and T.Padmapriya "Recent Trends In M2m Communications In $4 \mathrm{~g}$ Networks And Evolution Towards 5g", International Journal of Pure and Applied Mathematics, ISSN NO:1314-3395, Vol-115, Issue -8, Sep 2017.

[27] S.V. Manikanthan, T. Padmapriya "An enhanced distributed evolved node-b architecture in 5G tele-communications network" International Journal of Engineering \& Technology (UAE), Vol 7 Issues No (2.8) (2018) 248-254.March2018.

[28] S.V. Manikanthan, T. Padmapriya, Relay Based Architecture For Energy Perceptive For Mobile Adhoc Networks, Advances and Applications in Mathematical Sciences, Volume 17, Issue 1, November 2017, Pages 165-179 